\title{
MEJORANDO LA INTERPRETACIÓN DE SERIES TEMPORALES USANDO APRENDIZAJE NO SUPERVISADO
}

\section{IMPROVING THE INTERPRETABILITY OF DINSAR TIME SERIES USING UNSUPERVISED LEARNING}

\author{
Jaime Sánchez \\ Laboratorio de Topografía y Geomática, E.T.S.I. de Caminos, Canales y Puertos, Universidad Politécnica de Madrid, C/ Prof. \\ Aranguren, 3, Ciudad Universitaria, 28040 Madrid, España. jsanchez@detektia.com
}

\begin{abstract}
:
DInSAR technology provides high density of information about the temporal ground and infrastructure deformation rate with millimetre precision and without the need of ground instrumentation. DInSAR analyses the information within the radar phase and amplitude of a series of Synthetic Aperture Radar images. These images are acquired over large areas at the same location and at different dates to obtain the deformation time series on points showing low phase noise. It allows measuring surface movements and deformation with extensive area coverage, having lower cost per area compared to conventional techniques and the possibility of obtaining data before, during and after the event that is being studied. Therefore, DInSAR technology possesses great advantages over traditional technologies and could act in some cases a substitute for them for measuring millimetric ground and infrastructure movements. In many cases, the large volume of output points often difficult the manual inspection of the resultant dataset. Appropriate postprocessing methodologies are needed to properly interpret the data facilitating the objective interpretation of the results and the decision-making. This paper addresses this interpretation problems by 1 ) analysing the results of applying different data mining procedures to a case study in Madrid and 2) creating a systematic workflow useful for a preliminary analysis of the data. Principal Component Analysis is applied to reduce the dimensionality of the problem while minimizing the information loss and time series clustering and different unsupervised learning algorithms are used to classify the data based on similar characteristics.
\end{abstract}

Key words: geodesy, principal component analysis, interferometric synthetic aperture radar, DInSAR, unsupervised learning, clustering

\section{Resumen:}

La tecnología DInSAR provee una gran cantidad de información sobre la deformación a lo largo del tiempo del terreno y las infraestructuras con precisión milimétrica y sin la necesidad de usar instrumentación de campo. Durante el procesamiento DInSAR se analiza la información contenida en la fase y la amplitud de una serie de imágenes obtenidas mediante un Radar de Apertura Sintética. Estos cubrirán la misma área en distintos momentos para de este modo poder seleccionar los píxeles que poseen menor ruido en la fase permitiendo la medición de movimientos superficiales a lo largo de varios kilómetros cuadrados de área y de manera más económica que mediante métodos convencionales y teniendo además la posibilidad de obtener datos anteriores, contemporáneos o posteriores al evento estudiado. Por todo esto, el procesamiento DInSAR posee grandes ventajas sobre tecnologías tradicionales para medir deformaciones y movimientos milimétricos tanto del suelo como de infraestructuras. En muchos casos, el gran volumen de datos obtenido dificulta la inspección manual de estos. Lo que hace necesario el uso de metodologías apropiadas que simplifiquen la interpretación los datos facilitando así también la toma de decisiones. Este articulo intenta afrontar esos problemas 1) analizando los resultados de aplicar diferentes estrategias de minería de datos 2) creando un flujo de trabajo automatizado que facilite un análisis preliminar de los datos. Aplicando Análisis de Componentes Principales para reducir la dimensionalidad del problema y usando algoritmos de clusterización para agrupar los puntos por características similares.

Palabras clave: geodesia, análisis de componentes principales, radar de apertura sintética, DInSAR, aprendizaje no supervisado, clusterización

\section{Introducción}

El envejecimiento y el deterioro de infraestructuras es un problema global que provoca grandes pérdidas económicas debidas a la falta de mantenimiento preventivo y que, en último término, puede provocar la pérdida de vidas humanas. Las infraestructuras pueden modificar su comportamiento durante su vida útil, debido al deterioro de materiales, cambios en las cargas, condiciones ambientales, errores de diseño y construcción. Además, otros fenómenos y procesos, como terremotos, cambios del nivel o del flujo de agua subterránea por sobreexplotación acuíferos 0 la ejecución de grandes obras subterráneas en entornos urbanos pueden provocar la deformación de la superficie de la Tierra afectando de manera más o menos grave a las infraestructuras que se encuentran en superficie (Bischoff et al. 2020; Scoular et al. 2020).

Para prevenir, anticipar y reducir los daños y el deterioro de infraestructuras es esencial desarrollar sistemas que permitan detectar de forma temprana los movimientos y deformaciones milimétricas tanto de la superficie del 
terreno como de la infraestructura en sí. El sector de la ingeniería civil posee la necesidad clara de cuantificar el estado actual y futuro de todo tipo de infraestructuras en grandes áreas a través de la monitorización avanzada de su salud estructural. Los procedimientos más habituales para controlar movimientos y deformaciones en obras de ingeniería como son la topografía de precisión (nivelación), los inclinómetros, acelerómetros o el DGPS (Sistema de Posicionamiento Global Diferencial) no son económica o técnicamente viables en grandes áreas y, en la mayoría de los casos, no dan respuesta a las necesidades actuales de monitorización continua de grandes obras de ingeniería civil. En este contexto el desarrollo de nuevos sistemas eficientes de control y seguimiento de los movimientos milimétricos en infraestructuras que permitan analizar su estado, anticipar su comportamiento y generar un sistema de alerta temprana (García et al. 2020) son clave en un sector de tanta importancia.

Las técnicas basadas en interferometría radar satelital como el DInSAR son métodos que han avanzado de forma notable en los últimos años (Crosetto et al. 2016) y que permiten monitorizar masivamente movimientos milimétricos en la superficie del terreno e infraestructuras sin necesidad de instrumentación en tierra. Los cambios en la elevación y desplazamiento en planimetría de la superficie terrestre se registran mediante el tratamiento de la fase de la señal radar, utilizando la interferometría de pares de imágenes obtenidas mediante un Radar de Apertura Sintética (SAR) tomadas en la misma zona en distintas fechas, pudiendo llegar a conseguir la detección de movimientos con precisión milimétrica al trabajar con series temporales lo suficientemente largas. La tecnología DInSAR hace posible identificar puntos de respuesta estable o de "Persistent Coherence", los cuales pueden ser monitoreados sistemáticamente a lo largo del tiempo sobre grandes áreas e identificar deformaciones superficiales que ocurren en el terreno en orden de milímetros tanto en movimientos verticales, así como en desplazamientos horizontales.

La técnica DInSAR ha demostrado la capacidad de medir con éxito pequeños desplazamientos de estructuras con precisión milimétrica en muchos estudios científicos (Gascón et al. 2013; Herrera et al. 2013; Karila et al. 2005). La técnica DInSAR aplicada a la medición de la subsidencia superficial tiene varias ventajas, incluyendo una amplia cobertura de área dentro de una sola imagen (de miles de $\mathrm{km}^{2}$ ), menor coste por $\mathrm{m}^{2}$ en comparación con las técnicas convencionales, y la posibilidad de obtener datos antes, durante y después de la construcción o evento que se está estudiando (Tomás et al. 2014). Por lo tanto, la tecnología DInSAR es una tecnología emergente con grandes ventajas sobre las tecnologías tradicionales ( $\mathrm{y}$ en muchos casos un sustituto de ellas) para medir los movimientos milimétricos en obras e infraestructuras.

A pesar de todas estas ventajas que posee el DInSAR sobre los métodos geotécnicos y topográficos tradicionales, la correcta interpretación y análisis del gran volumen de información que genera esta tecnología es a día de hoy un reto importante al que se enfrenta el presente proyecto de investigación. El resultado del DInSAR son nubes de puntos densas sobre grandes áreas en las que cada punto describe la historia de las deformaciones en esa localización específica. La enorme cantidad de datos disponibles tanto en la dimensión espacial como temporal hacen que sea muy complicada la interpretación objetiva de la información. Es por ello, que la integración de técnicas que permitan mejorar la interpretación de los resultados y anticipar el comportamiento futuro del terreno e infraestructuras es clave para que esta tecnología ayude a la toma de decisiones tanto en la construcción de infraestructuras como en el diseño de las tareas preventivas y de mantenimiento.

\section{Procesado DinSAR}

El preprocesamiento de las imágenes SAR obtenidas con el sensor radar del satélite Envisat tendrá como objetivo corregistrar las imágenes para así seleccionar los píxeles que representan el mismo punto de la superficie de estudio y así poder crear después pares interferométricos.

La retrodispersión o retrorreflexión en la que se basa esta técnica es un fenómeno físico en el cual las ondas que inciden en un material en un ángulo determinado son reflejadas en el mismo ángulo, volviendo a la fuente que las produjo. De este modo, a partir del análisis de la fase contenida en los ecos que estos generen en el radar satelital obtendremos las series temporales de posiciones para los distintos reflectores que se encuentren en la zona, así como las velocidades medias para el periodo estudiado.

Existen dos grandes familias de algoritmos en función del tipo de retrorreflectores que usemos, los algoritmos PSDInSAR, que deben su nombre al uso de reflectores persistentes (Persistent Scatterers, Ferretti et al. 2001) que suelen ser píxeles individuales caracterizados por una ratio entre señal y ruido muy alto; este tipo de puntos es abundante en zonas urbanas donde existen gran cantidad de objetos estables con una alta reflectividad. Por otro lado, podemos usar un grupo de píxeles más ruidoso pero lo suficientemente homogéneo como para poder usar un análisis estadístico que nos permita recuperar información de la fase, estos grupos se conocen como reflectores distribuidos (Distributed Scatterers; Berardino et al. 2002) y son la base de los algoritmos DSInSAR, mucho más útiles para zonas con abundante vegetación. En nuestro caso se usará una técnica PSDInSAR denominada Interferometría de Retrorreflectores Persistentes (Persistent Scatterer Interferometry o PSI).

Uno de los mayores inconvenientes de esta forma de adquisición de datos es que las deformaciones medidas se encuentran en la dirección de la línea de visión del satélite, por lo que, para obtener la deformación vertical y horizontal del terreno, será necesario procesar dos geometrías de adquisición distintas (Fig. 1), ascendente cuando el satélite se mueve hacia el norte y descendente cuando lo hace hacia el sur, para después proyectar las deformaciones sobre los ejes deseados que en nuestro caso serán arriba-abajo y este-oeste.

\section{Aprendizaje no supervisado}

El aprendizaje no supervisado es una rama del aprendizaje automático que permite lidiar con datos no etiquetados (Kanevski et al. 2009). En nuestro caso, lo usaremos para generar áreas (agrupaciones de puntos) 
donde el comportamiento de la deformación del terreno o infraestructura es similar. Este tipo de procesos son muy útiles para detectar la dinámica general de movimiento de una zona amplia. Por otra parte, también puede servir como base para entrenar modelos detectores de anomalías que puedan ser usados como base para crear un sistema de alerta temprana ante catástrofes.

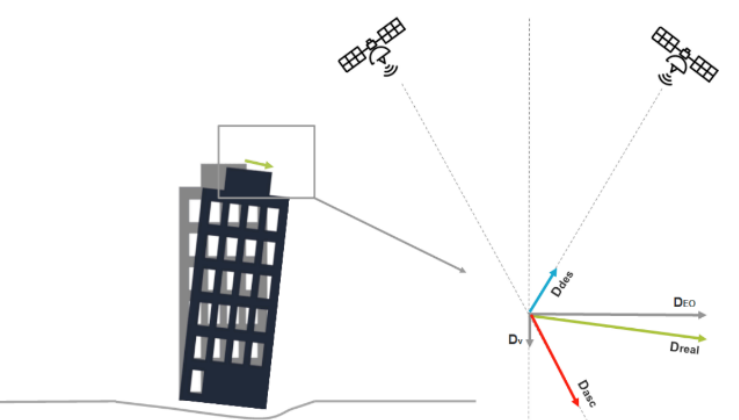

Figura 1: Esquema de la descomposición de movimientos a través de la combinación de geometría ascending y descending en movimientos en plano vertical y planimetría Este-Oeste.

Donde Dreal es el movimiento real del punto analizado, Ddes es el movimiento en LOS en geometría descending, Dasc es el movimiento en LOS en geometría ascending. Dv y Deo son los movimientos descompuestos en el eje vertical y en planimetría Este-Oeste.

En este trabajo se han explorado dos tipos de algoritmos de aprendizaje no supervisado usando como datos de partida las series temporales de deformación obtenidas con DInSAR, Análisis de Componentes Principales y clusterización. El primero de estos es un procedimiento utilizado para reducir la dimensión de los datos a la vez que se intenta minimizar la pérdida de información que ya ha sido incorporado con éxito en rutinas de procesamiento de datos DInSAR (Bonì et al. 2016) mientras que los algoritmos de clusterización son modelos capaces de dividir los puntos en un número dado de grupos en función de las características de estos.

\subsection{Análisis de Componentes Principales}

El algoritmo PCA se define como una transformación lineal capaz de expresar los puntos en una nueva base ortonormal donde la primera componente principal será el vector que maximiza la varianza de la proyección escalar del conjunto de datos.

La enésima componente principal se define a su vez como la que maximice la varianza al proyectar los puntos sobre su dirección y a su vez sea ortogonal a las $\mathrm{N}-1$ componentes anteriores (Jolliffe 2002).

En nuestro caso expresaremos las series temporales obtenidas como vectores $x_{-} i$ donde las coordenadas serán la deformación de ese punto en una fecha concreta, estos vectores compondrán las filas de una matriz $X$. De esta manera, la primera componente principal $w_{1}$ será un vector que cumpla la Ec. (1):

$w_{1}=\arg \max _{\|w\|=1}\left\{\sum_{i}\left(x_{-} i \cdot w\right)\right\}=\arg \max _{\|w\|=1}\left\{w^{T} X^{T} X w\right\}$

Mientras que las demás componentes se obtienen sustrayendo las componentes anteriores a $X$ (Ecs. 2 y 3 ):

$\hat{X}_{k}=X-\sum_{s=1}^{k-1} X w_{s} w_{s}^{T}$ $w_{k}=\arg \max _{\|w\|=1}\left\{\left\|\hat{X}_{k} w\right\|^{2}\right\}$

Una peculiaridad de esta transformación es que diagonaliza la matriz de covarianzas de los datos, ya que las componentes principales no poseen correlación entre sí.

\subsection{Algoritmos de clusterización}

Para la generación de los clústeres se ha utilizado $k$ means (Elkan 2003), este es un algoritmo basado en centroides que agrupa los datos en un número $k$ clústeres, donde cada uno de estos estará representado como un vector central y cada punto pertenece al clúster con el centro más cercano. El problema de optimización se define entonces como encontrar los centros de los clústeres que minimicen la distancia media cuadrada entre un punto y su clúster.

Al igual que en el apartado anterior, tomaremos los valores de las series temporales como las coordenadas que definen a cada punto. El cálculo de las distancias necesarias para la clasificación se realizará usando dos métricas distintas, comparando así la clasificación obtenida usando una distancia euclídea con la obtenida al asignar la distancia entre dos series temporales mediante el algoritmo de Dynamic Time Warping (DTW, Salvador and Chan 2007).

Este algoritmo se usa en análisis de series temporales como criterio para establecer la similitud entre dos series, para ello crea una correspondencia entre los puntos de una y otra serie (Fig. 2) para así poder identificar patrones similares en ambas series pese a que sucedan en momentos distintos y estén ligeramente distorsionados.
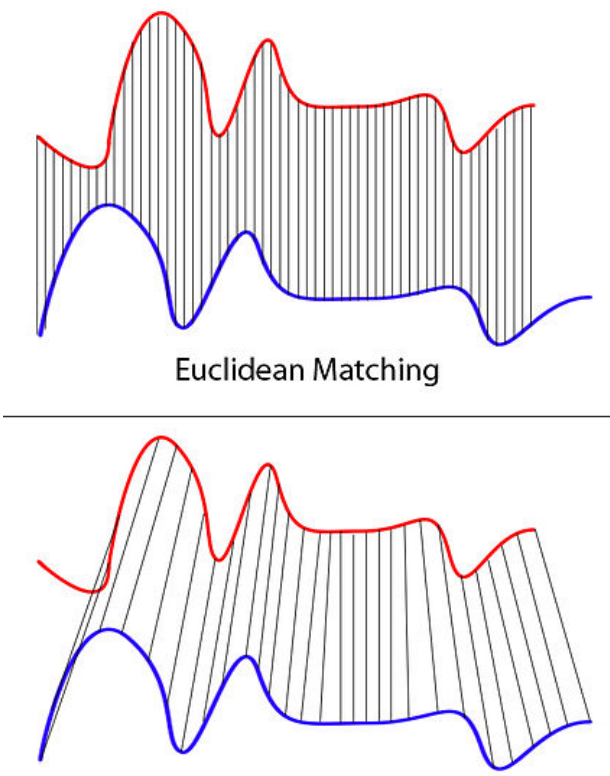

Dynamic Time Warping Matching

Figura 2: Diferencias entre la métrica euclídea y DTW aplicados a dos series temporales. La métrica euclídea será la suma de las diferencias al cuadrado para los valores de cada fecha de ambas series temporales mientras que con DTW se calculará una distancia basada en el emparejamiento óptimo de los valores de las series temporales. 
Para comparar el desempeño de los distintos modelos y los distintos parámetros, se calculará el error medio cuadrático cometido al clasificar las series, Ec. (4):

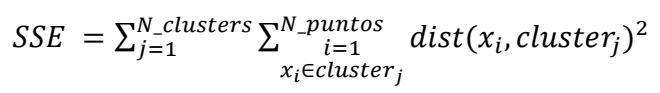

Donde $x_{i}$ será el vector que represente a la i-ésima serie temporal y cluster $r_{j}$ será el vector que contenga el centroide del j-ésimo clúster.

\section{Resultados}

La zona de estudio elegida se encuentra al Norte de Madrid (Fig. 3). Con una extensión de casi $500 \mathrm{~km}^{2}$, cubre un gran acuífero terciario detrítico sobre el que se encuentra gran parte de la zona metropolitana como el territorio al Noroeste de esta, donde se encuentran dos zonas de pozos cercanas a Fuencarral y Pozuelo de Alarcón, que presentan deformaciones cuasi elásticas debido a las fases de carga y descarga de los acuíferos (Ezquerro et al. 2014).

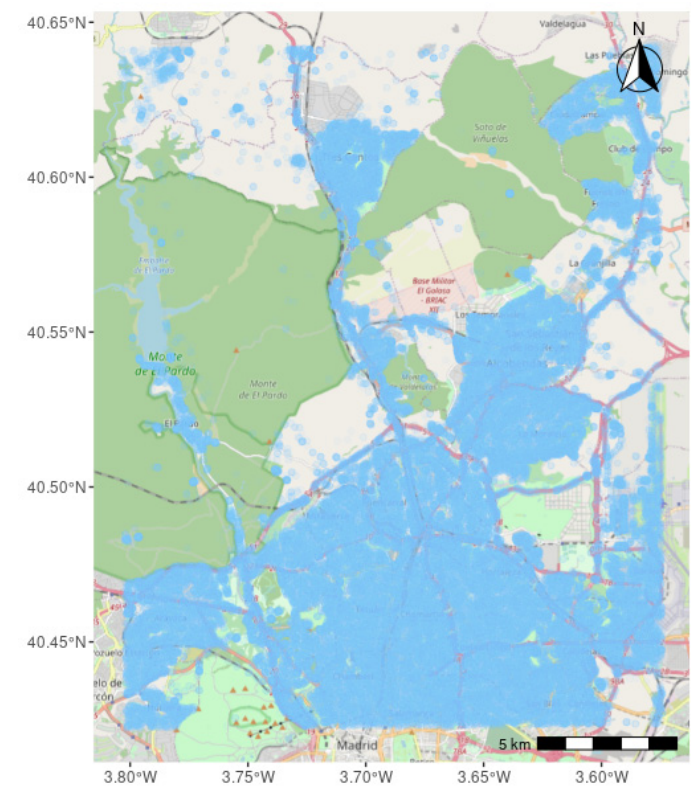

Figura 3: Zona de estudio que incluye parte del Norte de la zona metropolitana de Madrid. En azul se señalan los puntos que poseen serie temporal DInSAR.
Superpuestas al mapa se encuentran las localizaciones de los puntos pertenecientes al procesado PSDInSAR, realizado a partir de 31 imágenes ENVISAT tomadas con Advanced Synthetic Aperture Radar (ASAR) Image Mode (IM) entre 2003 y 2010. En total se han obtenido más de 100.000 puntos repartidos mayoritariamente por las zonas urbanas, debido a la mayor densidad de reflectores.

\subsection{Algoritmos de clusterización}

La relación entre el error SSE y el número de clústeres nos permite seleccionar de forma casi automática el número de clústeres idóneo para analizar los resultados DInSAR (Fig. 4) al realizar procesados con distinto número de clústeres (Figs. 5 y 6 ). Este suele establecerse en el codo de la curva, cuando el beneficio por aumentar el número de clústeres se reduce ampliamente, que será aproximadamente 4 en nuestro caso.

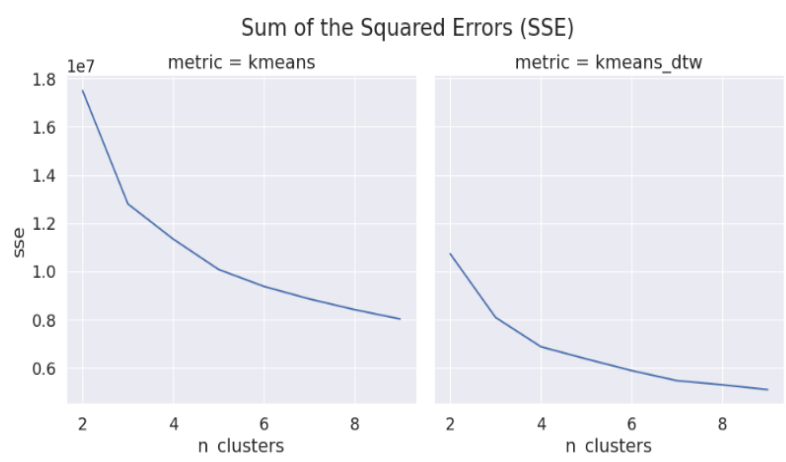

Figura 4: Suma de Errores Cuadrados para las clasificaciones desde 2 a 9 clústeres. Izquierda) Resultados para la métrica euclídea. Derecha) Resultados para DTW.

Los resultados (Figs. 7 y 8 ) muestran como las técnicas empleadas permiten separar, sin necesidad de disponer de información previa de la zona de estudio, los diferentes patrones de deformación del terreno presentes en el área analizada.

En el caso de estudio analizado no se observan diferencias importantes entre la utilización de la clasificación obtenida usando una distancia euclídea con la obtenida al asignar la distancia entre dos series temporales mediante el algoritmo de Dynamic Time Warping.

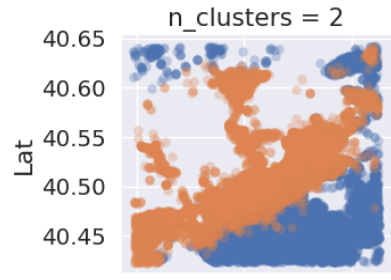

$\mathrm{n}$ clusters $=3$

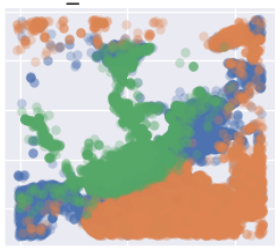

n clusters $=4$

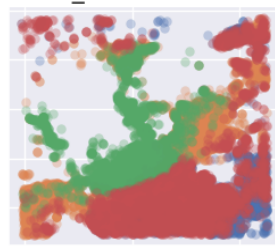

n_clusters $=8$
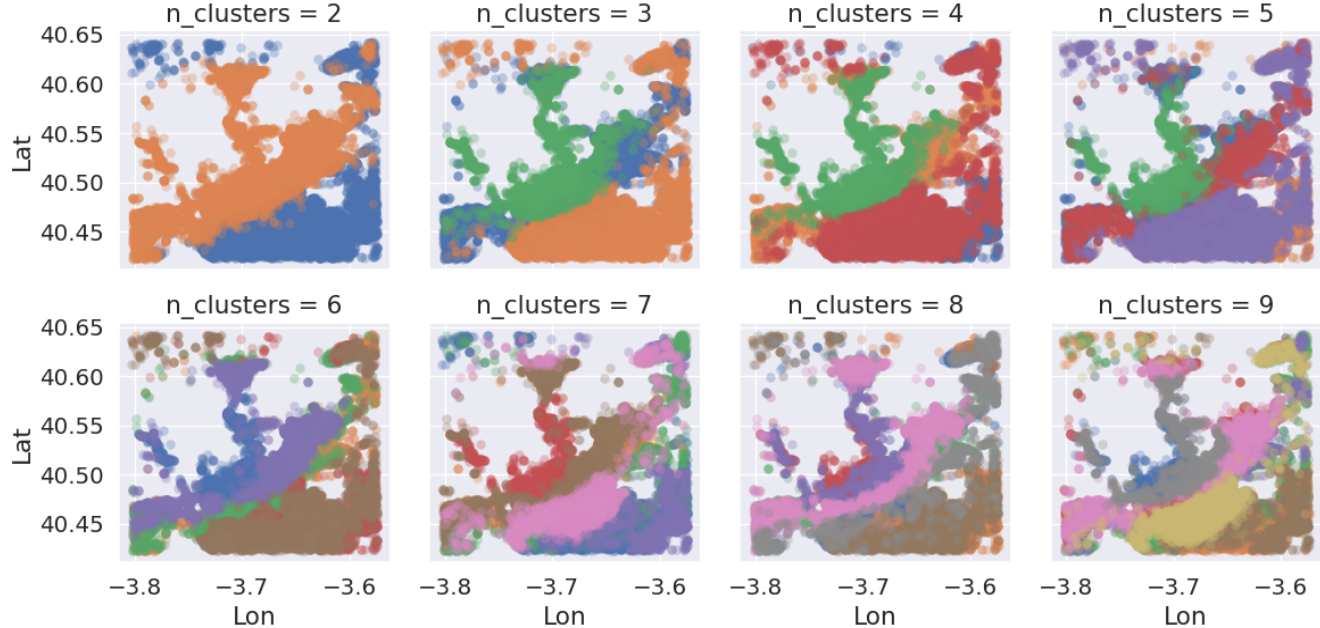

n_clusters $=9$

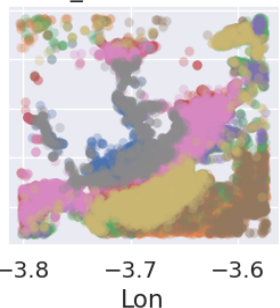

Lon

Figura 5: Clasificación de la zona de estudio utilizando el algoritmo kmeans con distinto número de clústeres y métrica euclídea para el cálculo de distancias. 

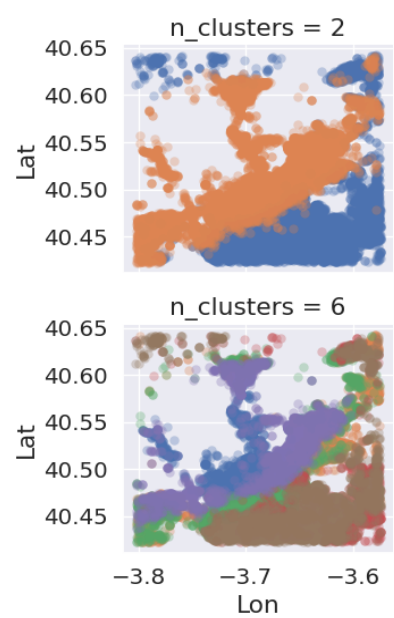

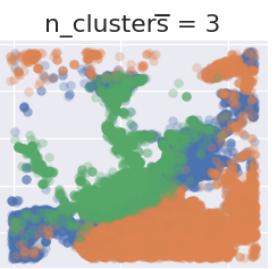

n_clusters $=7$

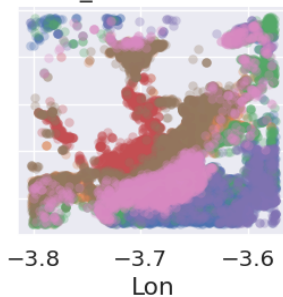

n clusters $=4$

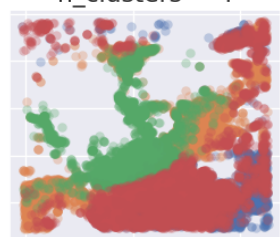

n_clusters $=8$

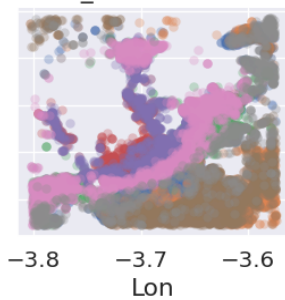

n_clusters $=5$

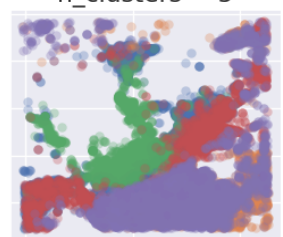

n_clusters $=9$

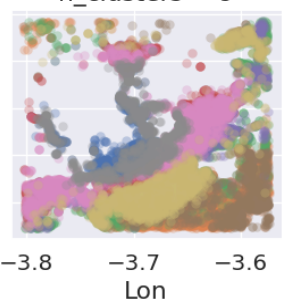

Figura 6: Clasificación de la zona de estudio utilizando el algoritmo kmeans con distinto número de clústeres y métrica DTW para el cálculo de distancias.
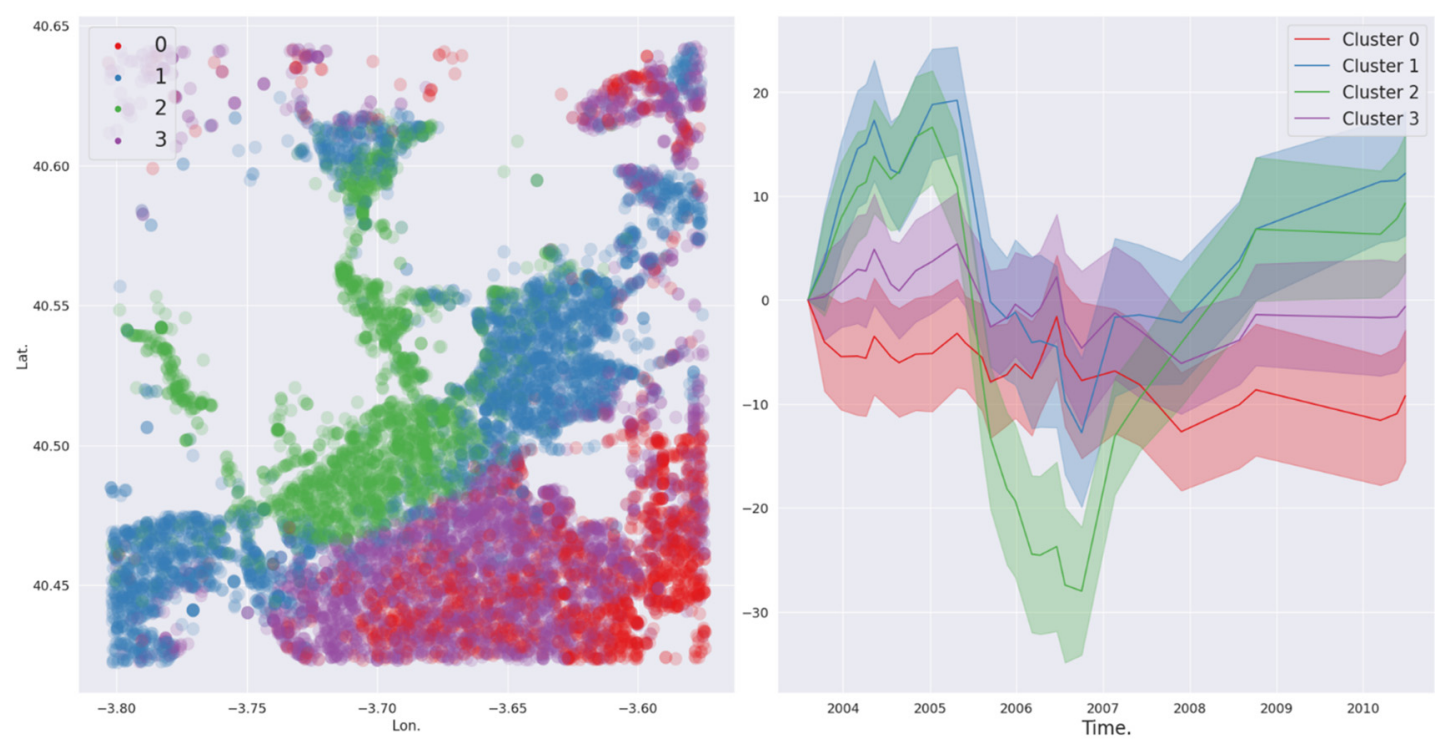

Figura 7: Clasificación de la zona de estudio utilizando el algoritmo kmeans con 4 clústeres y métrica euclídea para el cálculo de distancias, así como el comportamiento promedio de cada clúster. El clúster 2 agrupa los puntos más afectados por los ciclos de carga y descarga de los acuíferos y engloba las zonas de pozos de Fuencarral y Pozuelo de Alarcón.
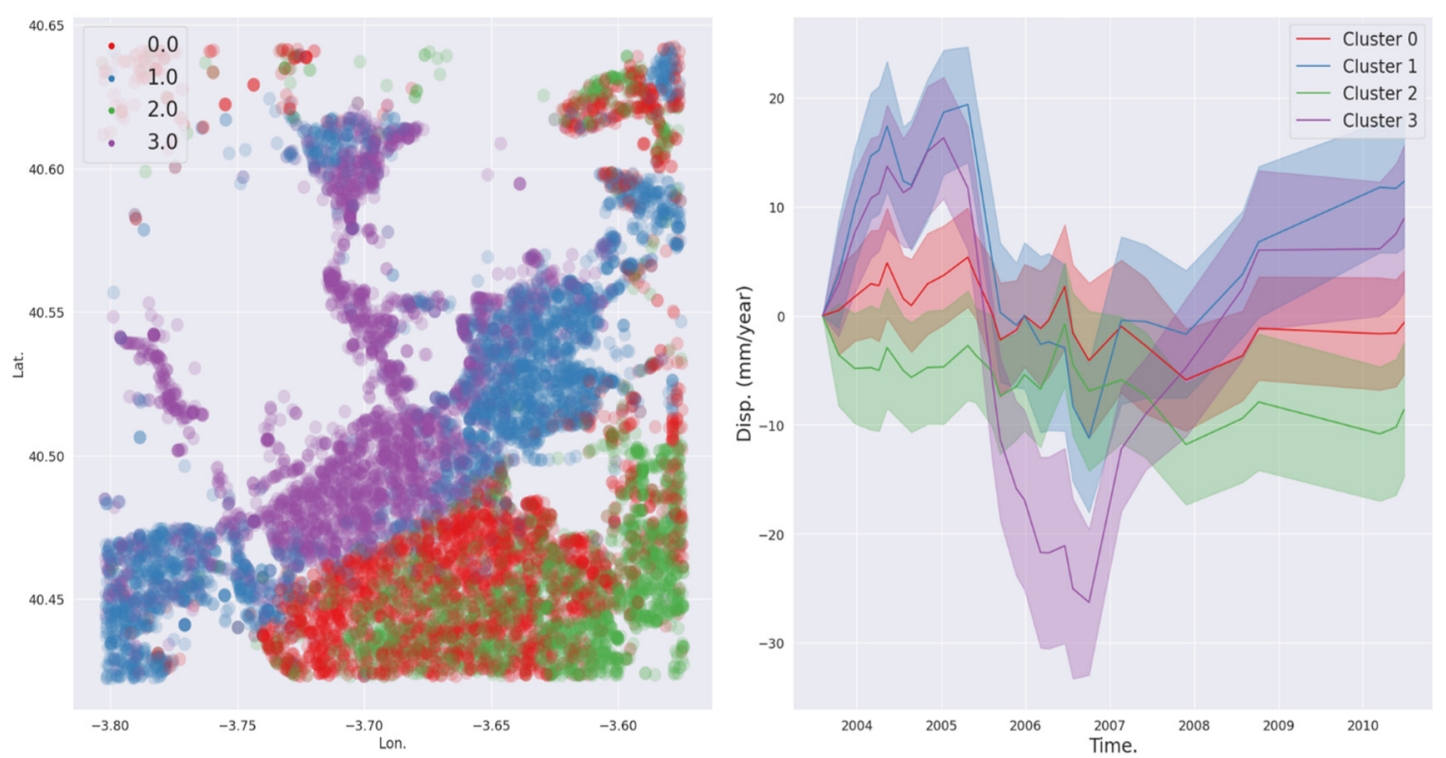

Figura 8: Clasificación de la zona de estudio utilizando el algoritmo kmeans con 4 clústeres y métrica DTW para el cálculo de distancias, así como el comportamiento promedio de cada clúster. El clúster 3 agrupa los puntos más afectados por los ciclos de carga y descarga de los acuíferos y engloba las zonas de pozos de Fuencarral y Pozuelo de Alarcón. 


\subsection{Análisis de Componentes Principales}

El único parámetro que nos permite elegir este algoritmo es el número de componentes principales que queremos elegir para representar las dimensiones de nuestros datos de salida.

Para poder valorar cuánta información aporta cada dimensión que añadimos, usaremos la varianza explicada por cada componente (Fig. 9). En ella se puede comprobar que, en nuestro caso, no tiene sentido utilizar más de dos componentes principales, puesto que las dos primeras componentes poseen más del $80 \%$ de la varianza de los datos y añadir las siguientes componentes no supone una mejora significativa.

En las Figuras 10 y 11 se puede visualizar a la izquierda, la aportación de cada punto a la primera y segunda componente principal mientras que, en la derecha se podrá ver la serie temporal que representa a dicha componente.

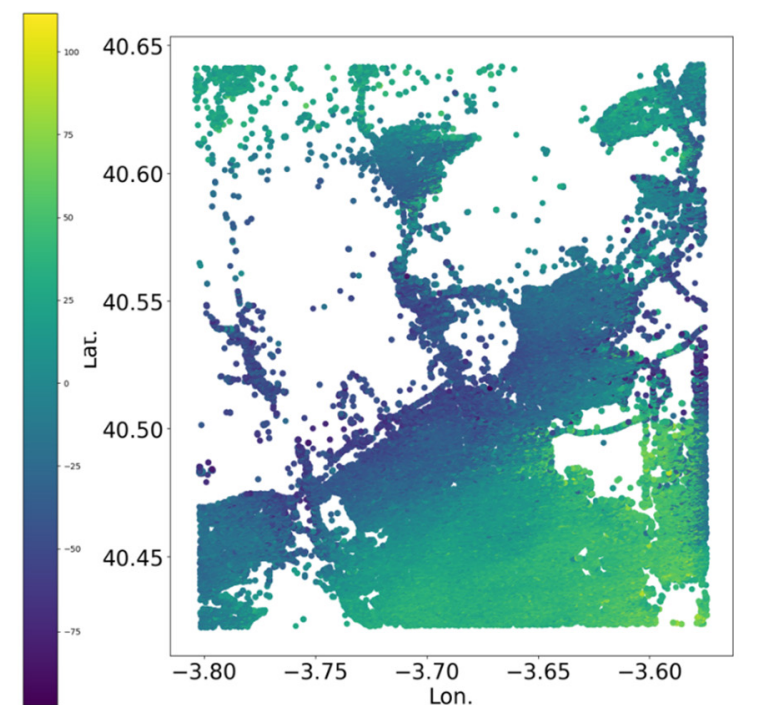

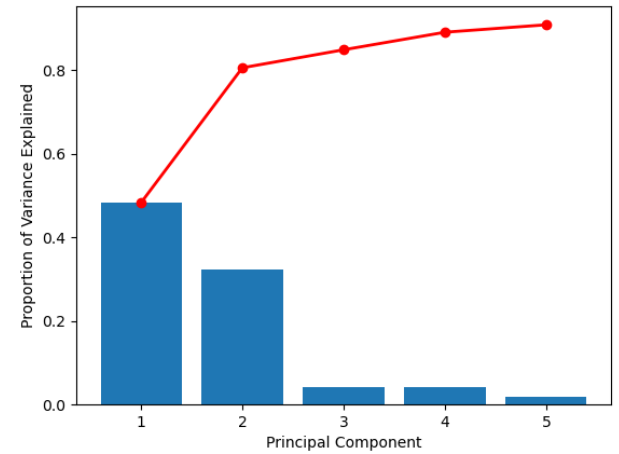

Figura 9: Gráfico de sedimentación de los resultados. Las barras representan el porcentaje de varianza explicada por cada componente principal y la línea es el valor cumulativo.

Los resultados de la aplicación de PCA sobre la base de datos de deformaciones DInSAR muestra también de forma visual, y sin necesidad de disponer información previa del área estudiada, el comportamiento del terreno (de levantamiento y subsidencia) en el periodo temporal analizado.

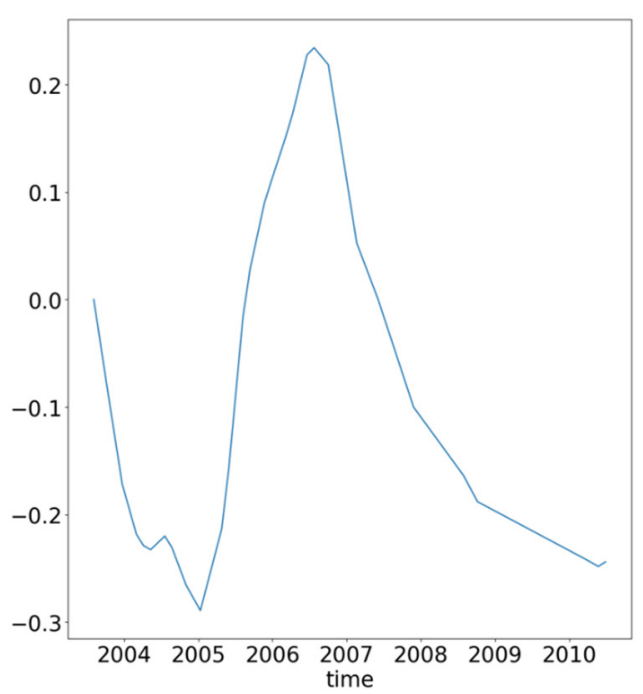

Figura 10: A la izquierda Coeficiente de cada punto en la primera componente principal mientras que, en la derecha se puede ver la serie temporal de desplazamientos de esta componente, calculada como la suma ponderada de todas las series temporales usando como peso el coeficiente asignado a cada punto.
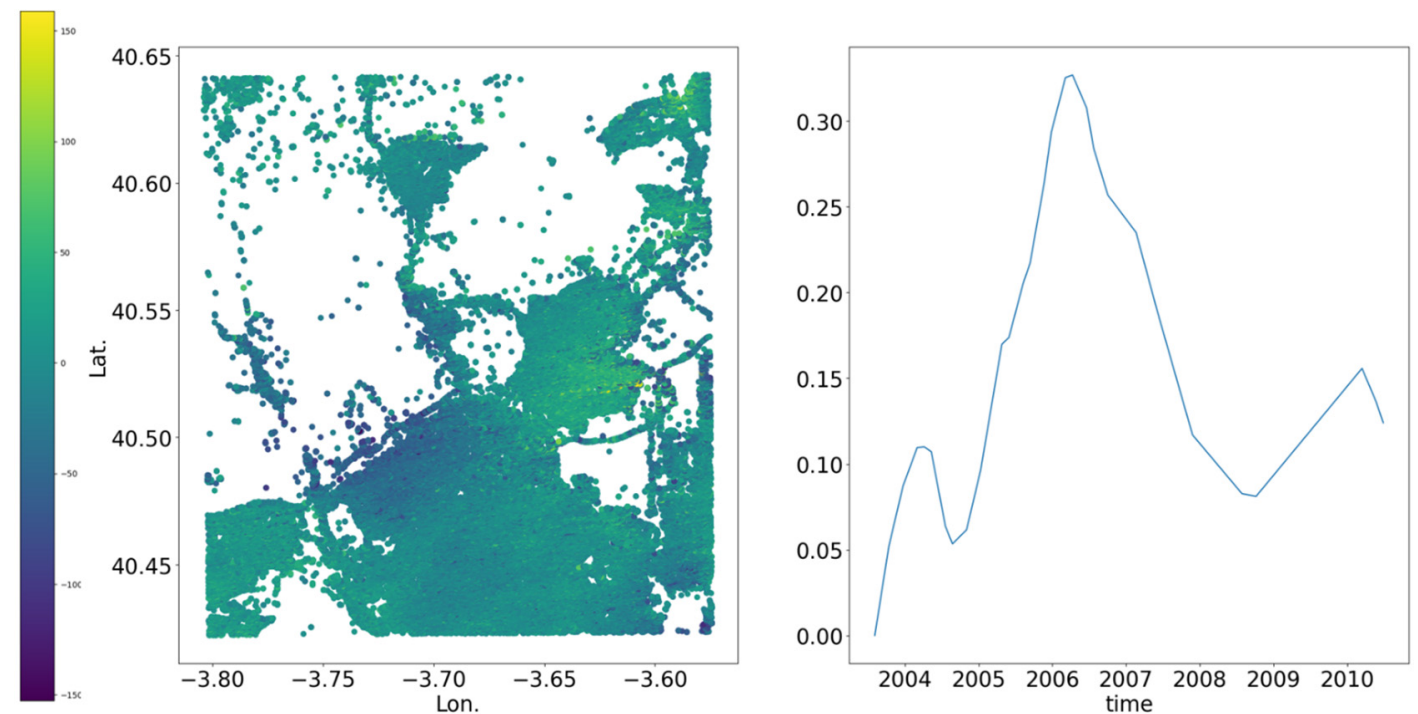

Figura 11: A la izquierda Coeficiente de cada punto en la segunda componente principal mientras que, en la derecha se puede ver la serie temporal de desplazamientos de esta componente, calculada como la suma ponderada de todas las series temporales usando como peso el coeficiente asignado a cada punto. 


\section{Discusión}

En este artículo se ha tratado de crear un procedimiento sistemático que facilite la interpretación de los datos obtenidos en procesados DInSAR, para ello se han utilizado dos procedimientos de aprendizaje no supervisado que apenas requieren ajuste a los hiperparámetros de los modelos.

Además, se ha podido comprobar la eficacia de estos métodos con un caso de uso real al poder extraer información del procesado sin necesidad de ningún tipo de información auxiliar.
No se han encontrado diferencias significativas entre las métricas usadas en la clusterización, en el futuro se intentará probar el sistema más distintos casos de estudio con comportamientos distintos en los que usar DTW suponga una ventaja.

En el caso del Análisis de Componentes Principales, se ha conseguido reducir notablemente la dimensión de los datos de 31 a 2, manteniendo gran parte de la información de los puntos, algo que se intentará usar como primera fase de distintos modelos de aprendizaje automático.

\section{References}

BERARDINO, P., G. FORNARO, R. LANARI, and E. SANSOSTI., 2002. A New Algorithm for Surface Deformation Monitoring Based on Small Baseline Differential SAR Interferograms. IEEE Transactions on Geoscience and Remote Sensing, 40(11), pp. 2375-2383. DOI: 10.1109/TGRS.2002.803792.

BISCHOFF, C. A., GHAIL, R. C., MASON, P. J., FERRETTI, A, and DAVIS, J. A., 2002. Revealing Millimetre-Scale Ground Movements in London Using SqueeSAR TM. Quarterly Journal of Engineering Geology and Hydrogeology, 53(1), pp. 311. DOI: $10.1144 / q j e g h 2018-075$.

BONì, R., PILLA, G., and MEISINA, C., 2016. Methodology for Detection and Interpretation of Ground Motion Areas with the A-DInSAR Time Series Analysis. Remote Sensing, 8, 686. DOI: 10.3390/rs8080686.

CROSETTO, M., MONSERRAT, O., CUEVAS-GONZÁLEZ, M., DEVANTHÉRY, N., and CRIPPA, B., 2016. Persistent scatterer interferometry: A review. ISPRS Journal of Photogrammetry and Remote Sensing, 115, pp. 78-89.

ELKAN, C., 2003. Using the triangle inequality to accelerate k-means. In Proceedings of the 20th international conference on Machine Learning ICML-03, pp. 147-153.

EZQUERRO, P., HERRERA, G., MARChAMALO, M., TOMÁS, R., BÉJAR-PIZARRO, M., and MARTíNEZ, R., 2014. A quasi-elastic aquifer deformational behavior: Madrid aquifer case study. Journal of Hydrology, 519, pp. 1192-1204. DOI: 10.1016/j.jhydrol.2014.08.040

FERRETTI, A., PRATI, C. and ROCCA, F., 2001. Permanent Scatterers in SAR Interferometry. IEEE Transactions on Geoscience and Remote Sensing, 39(1), pp. 8-20. DOI: 10.1109/36.898661.

GARCÍA, A. J., GONZÁLEZ-RODRIGO, B., MARTINEZ, S., MARTINEZ, R., and MARCHAMALO, M., 2021. Building health monitoring in the old town of Madrid: applicability of SAR Imagery to the monitoring of underground works through classification indexes. International Journal of Digital Earth, 14(3), pp. 271-287. DOI: 10.1080/17538947.2020.1815878

GASCÓN, B., BRU, G., CAMACHO, A. G., AVELLANEDA, M., PRIETO, J. F., GONZÁLEZ, P. J., HERRERA, G., TIAMPO, K. F., NAVARRO, J.A., and MORALES, 2013. Ladera Inestable en Leintz-Gatzaga. Estudio Geotécnico y Control del Deslizamiento con Técnicas Terrestres y Espaciales. Universidad Politécnica de Cataluña

GOLD, O., and SHARIR, M., 2018. Dynamic time warping and geometric edit distance: Breaking the quadratic barrier. ACM Transactions on Algorithms (TALG), 14(4), pp. 1-17. DOI: 10.1145/3230734

HERRERA, G., GUTIÉRREZ, F., GARCíA-DAVALILLO, J. C., GUERRERO, J., NOTTI, D., GALVE, J. P., FERNÁNDEZMERODO, J.A., and COOKSLEY, G., 2013. Multi-sensor advanced DInSAR monitoring of very slow landslides: The Tena Valley case study (Central Spanish Pyrenees). Remote Sensing of Environment, 128, pp. 31-43. DOI: 10.1016/j.rse.2012.09.020

HOOPER, A., ZEBKER, H., SEGALL, P., and KAMPES, B., 2004. A new method for measuring deformation on volcanoes and other natural terrains using InSAR persistent scatterers. Geophysical research letters, 31(23).

JOLLIFFE, I., 2002. Principal Component Analysis. Springer Series in Statistics. New York: Springer-Verlag. DOI: $10.1007 / \mathrm{b} 98835$.

KANEVSKI, M., POZDNUKHOV, A., and TIMONIN, V., 2008. Machine learning algorithms for geospatial data. Applications and software tools.

KARILA, K., KARJALAINEN, M., and HYYPPÄ, J., 2005. Urban land subsidence studies in Finland using synthetic aperture radar images and coherent targets. Photogramm. J. Finland, 19, pp. 43-53.

SALVADOR, S., AND CHAN, P., 2007. Toward accurate dynamic time warping in linear time and space. Intelligent Data Analysis, 11(5), pp. 561-580. 
SCOULAR, J., GHAIL, R., MASON, P., LAWRENCE, J., BELLHOUSE, M., HOLLEY, R., and MORGAN, T., 2020. Retrospective InSAR analysis of East London during the construction of the Lee Tunnel. Remote Sensing, $12(5), 849$. DOI: $10.3390 /$ rs12050849

TOMÁS, R., ROMERO, R., MULAS, J., MARTURIÀ, J. J., MALLORQUÍ, J. J., LOPEZ-SANCHEZ, J. M., HERRERA, G., GUTIÉRREZ, F., GONZÁlEZ, P. J., FERNÁNDEZ, J., DUQUE, S., CONCHA-DIMAS, A., COCKSLEY, G., CASTAÑEDA, C., CARRASCO D., and BLANCO P., 2014. Radar interferometry techniques for the study of ground subsidence phenomena: a review of practical issues through cases in Spain. Environmental earth sciences, 71(1), 163181. 